\title{
Madame Guyon, Correspondance. Tome III: Chemins mystiques
}

\section{Benedetta Papasogli}

\section{Q OpenEdition}

1 Journals

\section{Edizione digitale}

URL: http://journals.openedition.org/studifrancesi/30222

DOI: $10.4000 /$ studifrancesi.30222

ISSN: 2421-5856

\section{Editore}

Rosenberg \& Sellier

\section{Edizione cartacea}

Data di pubblicazione: 1 avril 2006

Paginazione: 149-150

ISSN: 0039-2944

\section{Notizia bibliografica digitale}

Benedetta Papasogli, «Madame Guyon, Correspondance. Tome III: Chemins mystiques», Studi Francesi

[Online], 148 (XLX | I) | 2006, online dal 30 novembre 2015, consultato il 21 avril 2021. URL: http:// journals.openedition.org/studifrancesi/30222 ; DOI: https://doi.org/10.4000/studifrancesi.30222

Questo documento è stato generato automaticamente il 21 avril 2021.

\section{(c) $(1) \&$}

Studi Francesi è distribuita con Licenza Creative Commons Attribuzione - Non commerciale - Non opere derivate 4.0 Internazionale. 


\title{
Madame Guyon, Correspondance. Tome III: Chemins mystiques
}

\author{
Benedetta Papasogli
}

\section{NOTIZIA}

MADAME GUYON, Correspondance. Tome III: Chemins mystiques, édition critique établie par Dominique Tronc, Paris, Honoré Champion, «Bibliothèque des correspondances, mémoires et journaux», 2005, pp. 934.

Con un nuovo volume di quasi mille pagine, continua a svolgersi il grandioso disegno editoriale che sta portando in luce massicci sommersi o poco noti dell'opera di Madame Guyon. Dopo gli scritti autobiografici, giunge ora a compimento la trilogia della Correspondance e già il curatore di questa edizione critica, Dominique Tronc, annuncia due successivi volumi che raccoglieranno opuscoli e trattati. Niente è semplice, oggi come ieri, in ciò che riguarda Madame Guyon, anche se molto vi è di affascinante nella sua vicenda e nel suo modo di raccontarla. Niente è semplice nemmeno nello schema editoriale seguito da Dominique Tronc, studioso infaticabile che, all'occorrenza, torna sul già fatto, con integrazioni e correzioni, come accade in questo tomo che chiude l'arco della corrispondenza della "dame directrice". Dopo le lettere di Directions spirituelles apparse nel 2003, dopo l'epistolario delle Années de combat edito nel 2004, il terzo volume ha un titolo aperto: Chemins mystiques. Esso preleva, dalla edizione settecentesca delle Lettres chrétiennes et spirituelles qui regardent la vie intérieure ou l'esprit du vrai christianisme, a cura di Pierre Poiret e poi di Jean- Philippe Dutoit, il grosso blocco delle lettere di cui sono incerti la data e il destinatario (integrato con 21 lettere dal Directeur mystique e con diversi Témoignages spirituels). Anche quando il destinatario si delinea, sia Fénelon o il marquis de Fénelon o Mlle de La Maisonfort, non viene del tutto meno l'aura caratteristica di questo volume: si stemperano qui i contorni netti delle situazioni storico-biografiche, si sfumano le circostanze che erano, negli altri tomi, ricostruibili come le scelte di un vasto dramma. Siamo fuori dalla controversia del puro amore, anche se gli estremismi del linguaggio della profetessa intrattengono sullo 
sfondo del testo un sospetto di tragedia. L'aria è rarefatta e pura: le lettere si identificano più per i temi che trattano che non per i volti che suscitano in controluce. Anonimo, indifferente alle rive che rispecchia, si svolge il grande fiume dell'insegnamento spirituale di Mme Guyon. La sua "sève" materna, che lei stessa paragona a un latte impetuoso, è libera di espandersi, tanto più che questo blocco di lettere spesso non datate si riferisce in genere agli ultimi anni della sua vita: al tramonto pacato in mezzo a una cerchia di discepoli cui continua a dire, da cuore a cuore, da "fondo" a "fondo" - moltiplicando l'espressione verbale di una comunicazione che pure non passa attraverso le parole - le cose di sempre.

Dominique Tronc (p. 11) fa suoi i criteri con cui erano state strutturate le edizioni di Poiret e Dutoit: «[...] pour y observer quelque sorte d'ordre, on a divisé chaque volume en trois parties, à la première desquelles on a rangé les lettres dont les sujets ont plus de rapport à l'état des commençants; à la seconde, celles qui regardent un état plus avancé; et à la troisième, les autres, qui désignent un progrès qui va encore plus loin» (notiamo la discrezione con cui si allude alla categoria dei «perfetti», che nel XVII secolo risultava ben problematica, e la sottolineatura posta sulla continuità piuttosto che sulle rotture nelle strade dell'anima). I "cammini mistici" riguardano dunque, in una accezione vasta, ogni stato di vita e ogni grado del progresso interiore. Nella raccolta di lettere abbiamo modo di verificare tanto il buon senso di Mme Guyon quanto il suo inquietante, persino stravagante ardimento spirituale, così come incontriamo a un tempo la monotonia di una scrittura trasandata, che nasce perpetuamente nel momento presente, senza revisione e senza previsione, e l'incanto di formule in cui l'acuità della coscienza si allea con la forza dell'immaginario.

Una volta ultimata la monumentale edizione della Corrispondenza di Fénelon, intrapresa, com'è noto, da Jean Orcibal e portata a termine da Jacques Le Brun e Irénée Noye, ecco che un altro pezzo del grande dossier relativo al "crepuscolo dei mistici" viene posta in luce adeguata. L'aggettivo "secret" è stato applicato più volte alla corrispondenza di Mme Guyon: segreto lo scambio epistolare con Fénelon, segrete altre sue relazioni non prive di rilievo storico... tra la ribalta e il segreto è trascorsa questa esistenza straordinaria, a cui non si può rendere migliore giustizia che quella di una conoscenza esaustiva. Gli apparati critici del volume, sobri e piuttosto tecnici, rinviano sostanzialmente alle introduzioni dei precedenti tomi per quanto riguarda una rilettura tematica della corrispondenza; ma un aspetto sta a cuore al curatore: ribadire la continuità fra Mme Guyon e una scuola di spirituali e di mistici francesi, fra cui Jean de Bernières e il Père Jean Chrysostome, che la spiegano ancor più di quanto non farebbe la rete d'influssi del quietismo europeo. Non a casa l'ultima "pièce" della raccolta è la strana lettera di una "paysanne" anonima, presa dalla follia della perdita, che parla di sé col timbro selvaggio che ha talora la voce di Surin o di Louise du Néant. La sapienza elitaria di un cenacolo spirituale cerca in tal modo il sua sigillo fuori da sé, nei tratti anonimi dell'"illettré éclairé", nei luoghi comuni - per quanto sconcertanti ed estremi di un secolo. 